Hier steht eine Anzeige.

Springer

\title{
Auflösung
}

\section{Purpura pigmentosa progressiva}

D ie Purpura pigmentosa progressiva (Morbus Schamberg) ist eine relativ häufige inflammatorische, hämorrhagisch-pigmentäre Hauterkrankung mit chronisch schubartigem Verlauf. Der Erkrankungsbeginn ist in jedem Alter möglich.

\section{Ätiologie}

Die genaue Ursache dieser chronischen oberflächlichen lymphozytären Vaskulitis mit Erythrozytendiapedese ist nicht geklärt. Mögliche Auslöser sind Medikamente, allen voran Schlaf- und Beruhigungsmittel wie Diazepam und Meprobamat. Aber auch Diuretika wie etwa Furosemid, das Retinoid Isotretinoin, Aspirin sowie etliche weitere Medikamente sind als mögliche Auslöser beschrieben.

Weitere Triggerfaktoren sind Nahrungsmittel, Nahrungsmittelzusatzstoffe sowie Kontaktallergene (vor allem Färbeund Bleichmittel in Textilien). Auch die chronisch venöse Insuffizienz (CVI) wird als auslösender Faktor diskutiert.

\section{Klinik}

Die Läsionen beginnen meist symmetrisch an den Unterschenkeln. Eine Ausbreitung auf den restlichen Körper ist möglich. Typisch sind unregelmäßig konfigurierte, unterschiedlich große, braun-rote Flecken mit umgebenden, teils cayennepfefferartigen Petechien, die diaskopisch nicht wegdrückbar sind. Die Oberfläche ist meist glatt, teils auch atrophisch. Seltener liegt eine feinlamelläre Schuppung mit ekzemartiger Morphe vor (eczematid-like Purpura nach Doucas und Kapetanakis).

Weitere klinische Sonderformen sind die lichenoide Purpura (GougerotBlum-Syndrom), der Lichen aureus sowie rein teleangiektatische Formen wie die Purpura anularis teleangiectodes nach Majocchi.

\section{Differenzialdiagnose}

Klinisch muss differenzialdiagnostisch an eine stauungsbedingte Purpura jaune d'ocre sowie an verschiedene Vaskulitiden wie Purpura Schönlein-Henoch oder leukozytoklastische Vaskulitis gedacht werden. Differenzialdiagnosen der ekzemartigen Läsionen sind unter anderem eine Mykosis fungoides sowie das Stauungsekzem. Die histologische Untersuchung ist hier richtungsweisend.

\section{Therapie}

Die Therapie ist meist unbefriedigend, insbesondere wegen des oft chronisch rezidivierenden Verlaufs und des langsamen Therapieansprechens. Bei der medikamenteninduzierten Form führt das Absetzen der entsprechenden Arzneimittel zu einem relativ raschen $\mathrm{Ab}$ klingen der Symptome. Auslösende Faktoren werden jedoch häufig nicht gefunden. Bei der CVI ist eine suffiziente Kompressionstherapie anzustreben.

Unter einer kurzzeitigen Lokaltherapie mit glukokortikoidhaltigen Externa blassen die Herde oftmals deutlich ab. Bei ausgedehntem Befall kommen systemische Glukokortikoide ausschleichend zum Einsatz. Der Versuch mit Bioflavinoiden zeigt manchmal gute Ansprechraten bei insgesamt guter Verträglichkeit (Rutosid $2 \times 50 \mathrm{mg} / \mathrm{d}$ plus Vitamin C $2 \times 500 \mathrm{mg} / \mathrm{d}$ ). Bei ausgedehnten Befunden kann auch eine Creme- oder Bade-PUVA hilfreich sein.

\section{Dr. med. Stephanie Gorbatsch} Dr. med. Benjamin Khan Durani Haut- und Laserzentrum Heidelberg Bergheimer Str. 56a

69115 Heidelberg 